\title{
Biventricular Thrombi Associated with Cardiac Systolic Dysfunction and Disseminated Intravascular Coagulation from Heat Stroke
}

\author{
Kohsaku Goto, ${ }^{1} \mathrm{MD}$, Taichi Masuda, ${ }^{1} \mathrm{MD}$, Junichi Ohashi, ${ }^{1} \mathrm{MD}$, \\ Yusuke Nakatsu, ${ }^{1}$ MD and Fumitaka Nakamura, ${ }^{2}$ MD
}

\begin{abstract}
Summary
A 58-year-old man with non-ischemic cardiomyopathy visited a hospital once a month after his first hospitalization for heart failure. Three months later, he presented with consciousness impairment and heat stroke. Blood tests showed multiple organ failure, and echocardiography revealed biventricular thrombi. After admission, intensive care was provided, and anticoagulation therapy was initiated. The echocardiographic findings in the third week confirmed the complete disappearance of thrombi. Biventricular thrombi associated with disseminated intravascular coagulation from heat stroke is rare. We report the case of a patient who was treated with anticoagulation therapy only, without surgical intervention.
\end{abstract}

(Int Heart J 2021; 62: 687-694)

Key words: Congestive heart failure, Ventricular thrombosis, Non-ischemic cardiomyopathy

I ntraventricular thrombus is occasionally observed on cardiac echography. Compared with right ventricular (RV) thrombus, left ventricular (LV) thrombus is much more frequent. Indeed, LV thrombus is often seen after myocardial infarction of the left anterior descending artery, and a previous study reported that LV thrombus occurred in $4 \%$ of patients with ST-elevation myocardial infarction. ${ }^{1)}$ In contrast, RV thrombus is rare and limited to specific diseases, such as arrhythmogenic right ventricular cardiomyopathy (ARVC), RV infarction, Ebstein anomaly, and Bechet disease. ${ }^{2)}$ Furthermore, biventricular thrombi are extremely rare, with only a few reports worldwide. Therefore, an obvious therapy for biventricular thrombi has not yet been proposed, and there are currently no American Heart Association (AHA) or European Society of Cardiology (ESC) guidelines.

Here, we report the case of a patient with reduced ejection fraction (EF) and disseminated intravascular coagulopathy (DIC) caused by heat stroke who went on to develop biventricular thrombi. The patient was subsequently treated with anticoagulation therapy without the need for surgery.

\section{Case Report}

A 58-year-old man visited a hospital on foot with the chief complaint of dyspnea on exertion. Based on several examinations such as electrocardiography (ECG), chest Xrays (CXR), and transthoracic echocardiography (TTE)
(Figures 1,2), he was diagnosed with congestive heart failure corresponding to New York Heart Association (NYHA) class III. After admission and initiation of the treatment, his symptoms of dyspnea soon improved and his body weight promptly decreased from $59.4 \mathrm{~kg}$ to 48.7 $\mathrm{kg}$. After discharge, both the CXR and N-terminal pro brain natriuretic peptide (NT-pro BNP) findings were gradually ameliorated (Supplemental Figure 1), and his cardiac function improved from EF 5.9\% to EF 30.7\% (Supplemental Figure 2). However, in the middle of summer, just 3 months after discharge, he was transported to our hospital by ambulance because he was unable to ingest more than water and milk for 3 weeks, which led to a deterioration in his level of consciousness.

The patient had never received any medical checkup or suffered from any disease other than congestive heart failure. Candesartan (6 mg/day), furosemide $(30 \mathrm{mg} /$ day $)$, spironolactone $(25 \mathrm{mg} / \mathrm{day})$ and esomeprazole $(20 \mathrm{mg} /$ day) had been prescribed since the first hospitalization. $\beta$ Blockers were initiated on an outpatient basis, but consciousness impairment lead to the suspension of the medications, as well as the initiation of $\beta$-blockers. He had smoked 20 cigarettes per day for the past 38 years, and he had consumed 3 or 4 cans of beer $(350 \mathrm{~mL} / \mathrm{can})$ per day for many years. He had no allergies and no family history of cardiac disease.

When he arrived at the hospital, his level of consciousness was Japan Coma Scale (JCS) -30 and Glasgow Coma Scale (GCS) E4V3M6. His blood pressure was

From the ${ }^{1}$ Department of Cardiology, Shioda Memorial Hospital, Chiba, Japan and ${ }^{2}$ Third Department of Internal Medicine, Teikyo University Chiba Medical Center, Chiba, Japan.

Address for correspondence: Kohsaku Goto, MD, Department of Cardiology, Shioda Memorial Hospital, 550-1 Kouri Nagara-machi, Chosei-gun, Chiba 297-0203, Japan. E-mail: kosh1107raku@gmail.com

Received for publication August 7, 2020. Revised and accepted November 30, 2020

Released in advance online on J-STAGE May 15, 2021.

doi: 10.1536/ihj.20-558

All rights reserved by the International Heart Journal Association. 
A

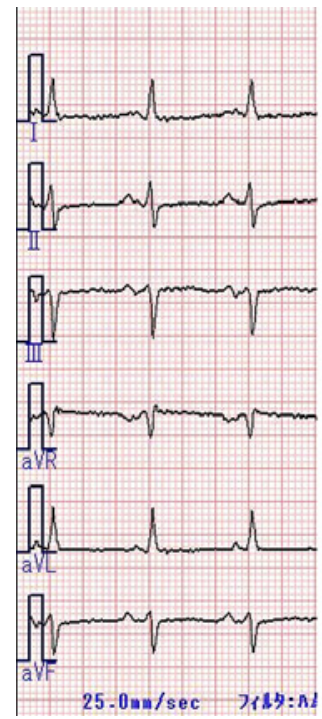

B

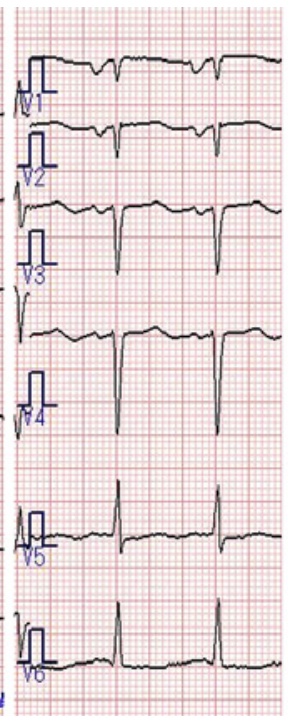

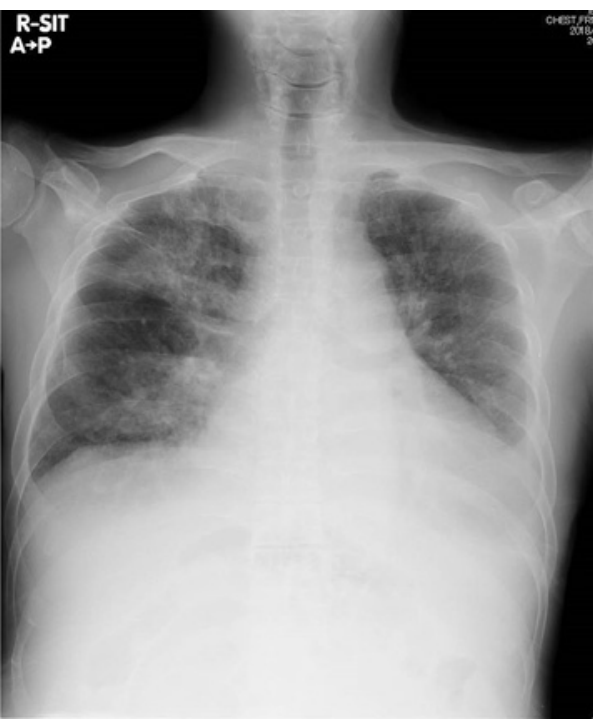

Figure 1. ECG and chest X-ray (CXR) findings at the first admission. A: ECG showed regular sinus rhythm, left high voltage and left axis. B: CXR revealed bilateral pulmonary congestion and pleural effusion.

A
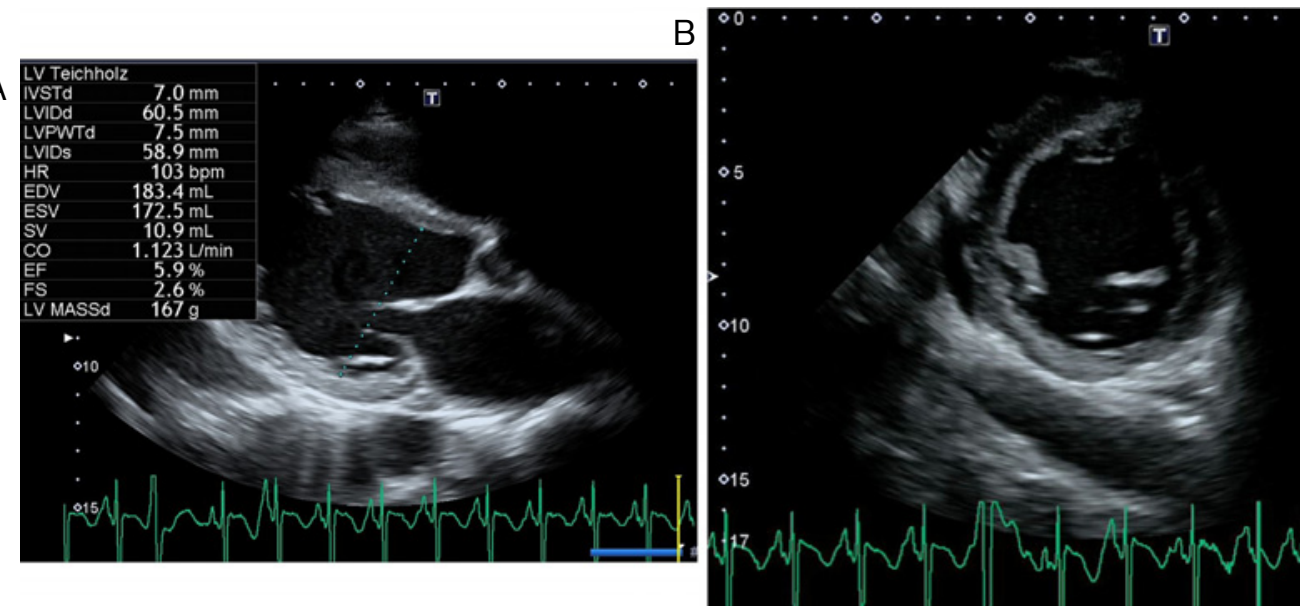

Figure 2. TTE finding at the first admission. A: Long axis view of TTE showed significant systolic dysfunction LV intraventricular diastolic/systolic diameter was $60.5 \mathrm{~mm} / 58.9 \mathrm{~mm}$. Ejection fraction was $5.9 \%$. B: Short axis view reveals pericardial effusion. The diameter of pericardial effusion at the posterior wall was $3.9 \mathrm{~mm}$ in diastole and 7.5 $\mathrm{mm}$ in systole.

148/118 $\mathrm{mmHg}$, his heart rate was $96 \mathrm{bpm}$, his oxygen saturation was $97 \%\left(\mathrm{O}_{2}\right.$ nasal cannula $3 \mathrm{~L} /$ minute), his respiratory rate was $36 /$ minute, and his body temperature was $37.2^{\circ} \mathrm{C}$. His height was $168 \mathrm{~cm}$, and his body weight decreased from $48.7 \mathrm{~kg}$ to $40.2 \mathrm{~kg}$ in 3 months. His extremities were dry and cold, cyanosis was prominent, and both dry tongue and lack of skin turgor were observed. Inspiratory and expiratory wheezes were not observed, and there was no evidence of lower leg edema. Arterial blood gas revealed respiratory alkalosis and compensated metabolic acidosis, in addition to high anion gap metabolic acidosis due to lactate accumulation. Blood tests indicated multiple organ failure and DIC (Table). An ECG revealed new inverted $\mathrm{T}$ waves in $\mathrm{I}, \mathrm{aVL}$, and the $\mathrm{V} 5$ and
V6 leads. CXR showed cardiac enlargement, but with no evidence of pulmonary congestion or pleural effusion (Figure 3). TTE revealed biventricular thrombi, in addition to systolic dysfunction and LV enlargement. Specifically, 3 ventricular thrombi $(27.8 \mathrm{~mm}$ long and $22.8 \mathrm{~mm}$ wide, $17.4 \mathrm{~mm}$ long and $11.9 \mathrm{~mm}$ wide, $23.8 \mathrm{~mm}$ long and 14.3 $\mathrm{mm}$ wide) were seen in the left ventricle, and two ventricular thrombi (12.7 $\mathrm{mm}$ long and $10.2 \mathrm{~mm}$ wide, 15.6 $\mathrm{mm}$ long and $10.6 \mathrm{~mm}$ wide) were observed in the right ventricle (Figure 4). Not only LV but also RV function declined, and the right ventricle tended to be dilated. Tricuspid regurgitation (TR) was mild, and the TR pressure gradient was $49 \mathrm{mmHg}$. None of the thrombi were mobile and were characterized as having a hyperechoic structure. 
Table. Laboratory Data of Second Admission

\begin{tabular}{|c|c|c|c|}
\hline Item & & Item & \\
\hline $\mathrm{pH}$ & 7.566 & WBC & $17,000 / \mu \mathrm{L}$ \\
\hline $\mathrm{pCo} 2$ & $13.3 \mathrm{mmHg}$ & Neu & $83.5 \%$ \\
\hline pO2 & $75 \mathrm{mmHg}$ & Ly & $12.8 \%$ \\
\hline $\mathrm{BE}$ & -10 & Mono & $3.5 \%$ \\
\hline $\mathrm{HCO} 3$ & 12.1 & Eos & $0.1 \%$ \\
\hline \multirow[t]{2}{*}{$\mathrm{AG}$} & 24.1 & Baso & $0.1 \%$ \\
\hline & & $\mathrm{RBC}$ & $55,270,000 / \mu \mathrm{L}$ \\
\hline ALB & $3.1 \mathrm{~g} / \mathrm{dL}$ & HGB & $17.5 \mathrm{~g} / \mathrm{dL}$ \\
\hline BUN & $105.6 \mathrm{mg} / \mathrm{dL}$ & HCT & $52.1 \%$ \\
\hline CRNN & $2.49 \mathrm{mg} / \mathrm{dL}$ & $\mathrm{MCV}$ & $94.4 \mathrm{fl}$ \\
\hline UA & $20.3 \mathrm{mg} / \mathrm{dL}$ & $\mathrm{MCH}$ & $31.7 \mathrm{pg}$ \\
\hline T-Bil & $5.78 \mathrm{mg} / \mathrm{dL}$ & $\mathrm{MCHC}$ & $33.6 \%$ \\
\hline $\mathrm{T}-\mathrm{CHO}$ & $196 \mathrm{mg} / \mathrm{dL}$ & PLT & $102,000 / \mu \mathrm{L}$ \\
\hline TG & $144 \mathrm{mg} / \mathrm{dL}$ & & \\
\hline LDL-CHO & $131 \mathrm{mg} / \mathrm{dL}$ & $\mathrm{TSH}$ & $4.203 \mu \mathrm{LU} / \mathrm{mL}$ \\
\hline $\mathrm{LDH}$ & $1344 \mathrm{U} / \mathrm{L}$ & free $\mathrm{T} 3$ & $0.89 \mathrm{pg} / \mathrm{mL}$ \\
\hline AST & $626 \mathrm{U} / \mathrm{L}$ & free $\mathrm{T} 4$ & $0.57 \mathrm{ng} / \mathrm{mL}$ \\
\hline ALT & $402 \mathrm{U} / \mathrm{L}$ & & \\
\hline ALP & $274 \mathrm{U} / \mathrm{L}$ & APTT & 52.1 seconds \\
\hline$\gamma \mathrm{GTP}$ & $84 \mathrm{U} / \mathrm{L}$ & PT-INR & 2.22 \\
\hline $\mathrm{CK}$ & $12546 \mathrm{U} / \mathrm{L}$ & D-dimer & $29.4 \mu \mathrm{g} / \mathrm{mL}$ \\
\hline CK-MB & $93 \mathrm{U} / \mathrm{L}$ & & \\
\hline NA & $146 \mathrm{mEq} / \mathrm{dL}$ & NT-proBNP & $>40,000 \mathrm{pg} / \mathrm{mL}$ \\
\hline $\mathrm{K}$ & $5.8 \mathrm{mEq} / \mathrm{dL}$ & & \\
\hline CRP & $1.81 \mathrm{mg} / \mathrm{dL}$ & & \\
\hline
\end{tabular}

Blood gas demonstrates respiratory alkalosis, compensated with metabolic acidosis, in addition, high anion gap indicated lactate acidosis due to circulatory failure. Renal impairment, the elevation of hepatobiliary enzymes and the collapse of mineral balance were also observed. The prolongation of prothrombin time-international normalized ratio (PT-INR) and the deterioration of platelet indicated DIC.

In plane computed tomography (CT), a low-density area was observed in each ventricle, which indicated biventricular thrombi (Figure 5), and no obvious findings supporting malignancy were obtained. We were unable to determine whether pulmonary embolism occurred due to the use of non-contrast CT. Ultrasonographic examination of the deep vein in the lower extremities showed no thrombus above the knee. Head magnetic resonance imaging (MRI) also revealed right hemisphere cerebral infarction, specifically, high signal in diffusion weighted imaging (DWI) and low signal in apparent diffusion coefficient (ADC) maps were consistent with acute lacunar infarctions (Figure 6).

After admission, intravenous fluids were administered to ensure peripheral perfusion, and an inotropic agent (dopamine) at $2 \mu \mathrm{g} / \mathrm{kg} /$ minute was also administered. For biventricular thrombi, anticoagulation with heparin was initiated. Adequate fluids were given, and his peripheral circulation gradually improved with no deterioration in respiration. Furosemide (40 mg/day) was restarted from the 2nd hospital day; his hemodynamic status was continuously stable, and his consciousness gradually ameliorated. No neurological disability was found after the improvement of his consciousness. Dopamine was tapered and terminated on the 7 th hospital day, and furosemide was switched from intravenous ( $40 \mathrm{mg} /$ day) to oral intake (20 $\mathrm{mg} /$ day) on the 10th hospital day. In the continuous observation of TTE, the biventricular thrombi tended to dimin- ish. The echocardiographic findings of the 3rd and 7th hospital days are shown in Figure 7; these thrombi remained immobile, and the echoic brightness was still high. The complete disappearance of the biventricular thrombi was finally confirmed on the 21st hospital day. During hospitalization, modification of warfarin was performed adjunctively with heparin. The D-dimer level was $29.4 \mu \mathrm{g} / \mathrm{mL}$ on admission but decreased to $2.1 \mu \mathrm{g} / \mathrm{mL}$ by the 4th week. His NT-pro BNP also improved from more than $40,000 \mathrm{pg} / \mathrm{mL}$ to $1,930 \mathrm{pg} / \mathrm{mL}$. His cardiac function was much improved in the TTE before discharge compared to the initial finding. To be more specific, the EF improved from $5.9 \%$ to $26 \%$, and the intraventricular diastolic/systolic diameter (Dd/Ds) decreased from $60.5 \mathrm{~mm} /$ $58.9 \mathrm{~mm}$ to $54.4 \mathrm{~mm} / 47.4 \mathrm{~mm}$. The patient required a certain period of time for rehabilitation, and he was discharged to his home on foot on the 66th day.

After discharge, the patient returned to the hospital regularly. The result of coronary CT revealed some degree of calcification of the 3 coronary vessels, but there were no signs of severe stenosis. The TTE findings 1 year after discharge revealed further improvement of the systolic function; the EF was ameliorated to $45 \%$, and the $\mathrm{Dd} / \mathrm{Ds}$ was $51 / 39 \mathrm{~mm}$. Furthermore, the inverted $\mathrm{T}$ waves observed in I, aVL, and leads V5 and V6 in the ECG at the time of the second admission reversed to normal 1 year after discharge (Supplemental Figure 3). The patient has continued to take his prescribed medications.

\section{Discussion}

Herein, we describe the case of biventricular thrombi in a patient with cardiac systolic dysfunction and DIC from heat stroke. There are several reports about biventricular thrombi in patients with systolic dysfunction, but, to the best of our knowledge, there have been no reports of cases combined with DIC from heat stroke. Thus, it is valuable to report that DIC could formulate biventricular thrombi in patients with systolic dysfunction.

When considering the cause of thrombus formation, Virchow's triad should be remembered, in which it is said that stasis, vessel wall injury, and hypercoagulability are the 3 most important factors for thrombosis. ${ }^{3)}$ In this case, the patient possessed 2 components of stasis and hypercoagulability. As for stasis, circulatory failure was induced by both systolic dysfunction and heat stroke. Although the blood pressure at the second admission was maintained, the blood test showed a significant elevation of lactate, which indicated anaerobic metabolism in the peripheral tissues. The mismatched oxygen supply for the demand of peripheral tissues exactly signifies circulatory failure. In the current case, circulatory failure was sufficient to cause congestion or stasis as a result of cardiac dysfunction, but the intravascular dehydration induced by heat stroke exacerbated the condition. Thus, systolic dysfunction and heat stroke were combined to trigger circulatory failure, which contributed to stasis. According to a previous report, ${ }^{4)}$ heat stroke could cause myocardial injury regardless of the underlying disease such as cardiomyopathy, but the pathophysiology of cardiac dysfunction in heat stroke is not fully understood yet. The pathologic study of victims of 
A

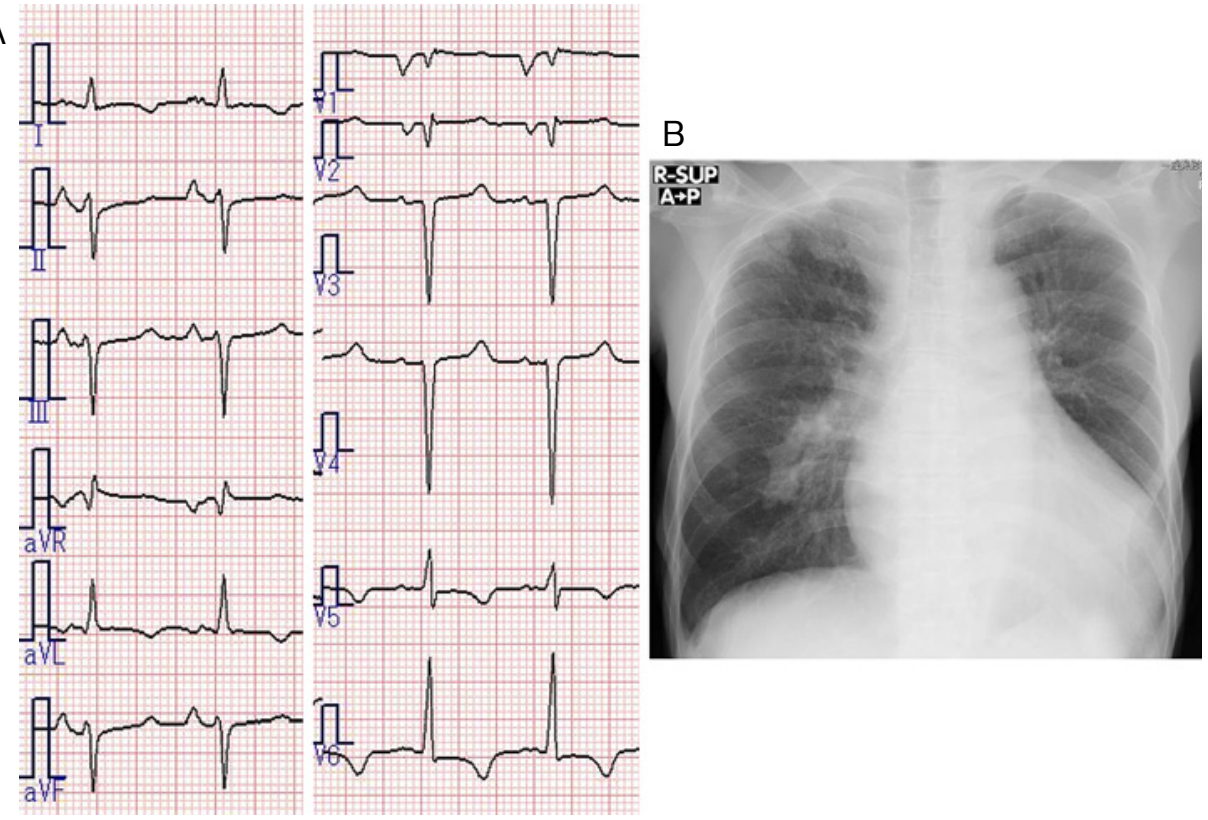

Figure 3. ECG and chest X-ray findings at the second admission. A: Inverted $\mathrm{T}$ waves in $\mathrm{I}$, aVL and the V5 and V6-leads newly emerged in comparison to the initial admission. B: Significant cardiomegaly remained, but there were no findings of congestion or bilateral pleural effusion.

\section{A}

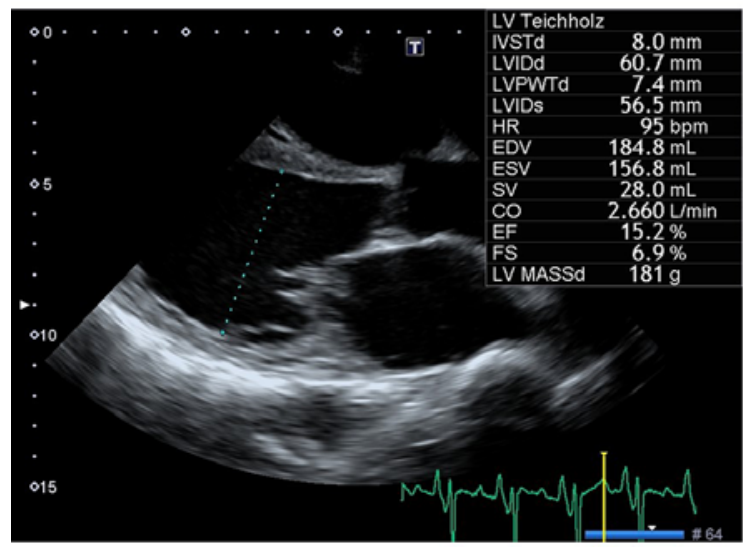

B

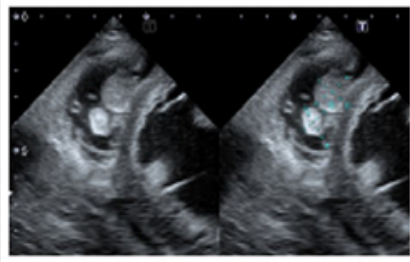

$\mathrm{RV}: 12.7^{*} 10.2 \mathrm{~mm}$ $15.6 * 10.6 \mathrm{~mm}$
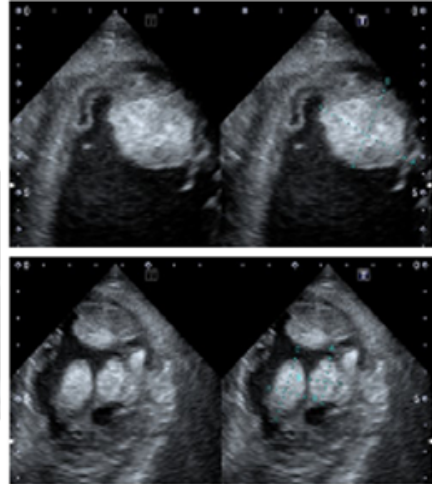

$\mathrm{LV}: 27.2 * 22.8 \mathrm{~mm}$

\section{$17.4 * 11.9 \mathrm{~mm}$}

Figure 4. TTE finding and biventricular thrombi at the second admission. A: Long axis view showed that LV intraventricular diastolic/systolic diameter was $60.7 \mathrm{~mm} / 56.5 \mathrm{~mm}$ and EF was $15.2 \%$. B: There were 5 biventricular thrombi in the apical portion of the biventricle. Three of them were found in the left ventricle $(27.2 \mathrm{~mm}$ long and $22.8 \mathrm{~mm}$ wide, $17.4 \mathrm{~mm}$ long and $11.9 \mathrm{~mm}$ wide, $23.8 \mathrm{~mm}$ long and $14.3 \mathrm{~mm}$ wide, respectively). The others were observed in the right ventricle ( $12.7 \mathrm{~mm}$ long and $10.2 \mathrm{~mm}$ wide, $15.6 \mathrm{~mm}$ long and $10.6 \mathrm{~mm}$ wide, respectively). These thrombi were immobile and showed hyperechoic brightness.

heat stroke revealed subendocardial hemorrhage and severe degeneration of cardiac muscle. Several proposed mechanisms of myocardial injury by heat stroke include an elevated plasma lipopolysaccharide concentration from portal circulation, estrogen deficiency, or increased levels of serum catecholamines. ${ }^{5)}$ In the current case, the main cause of cardiac dysfunction was not heat stroke because systolic function had already deteriorated before the second hospitalization, but these mechanisms could partially involve the exhibition of cardiac dysfunction. Surely, the blood pressure was maintained at the second admission; however, it is important to indicate that maintained blood pressure did not necessarily exclude intravascular dehydration from heat stroke since the diagnostic criteria of heat stroke do not include the deterioration of blood pressure. DIC occurred simultaneously with stasis, which led to a state of hypercoagulability, based on the fact that the Japanese Association for Acute Medicine (JAAM) acute 

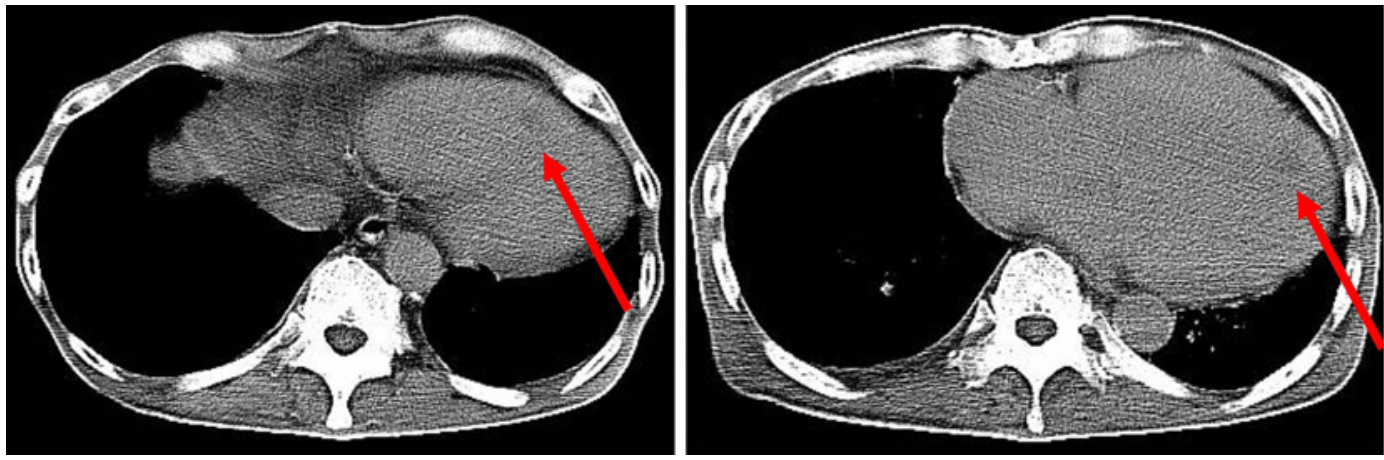

Figure 5. The plain CT finding at the second admission. Axial images of plain CT which indicates the existence of biventricular thrombi. The low density areas shown by red arrows suggest thrombi.
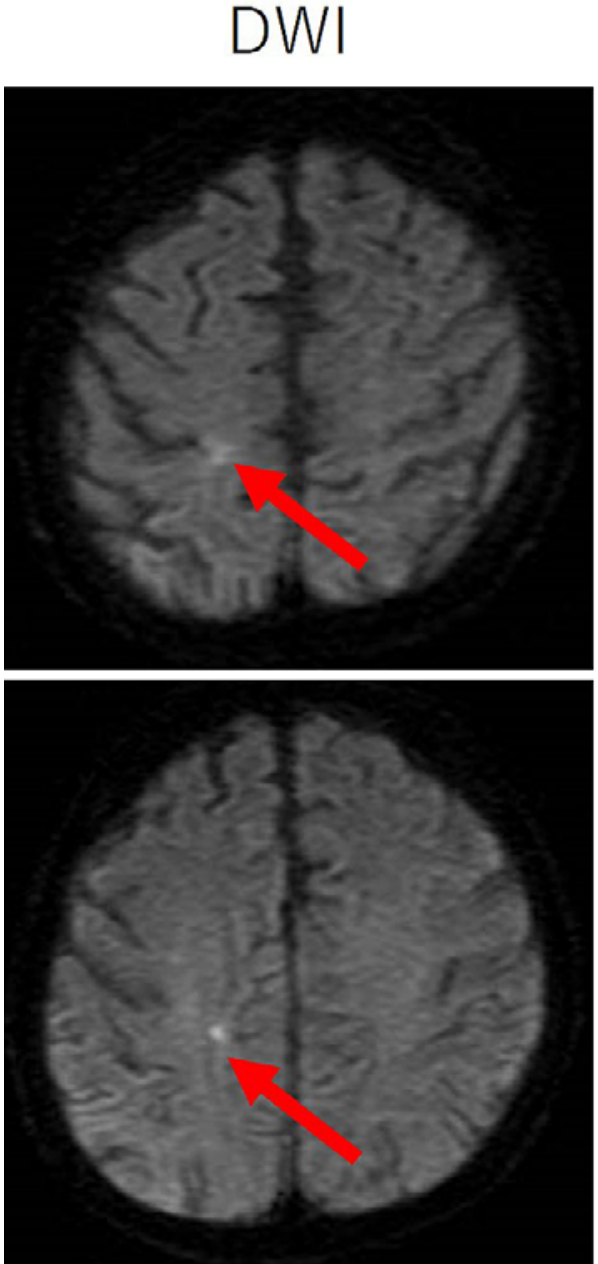

ADC map
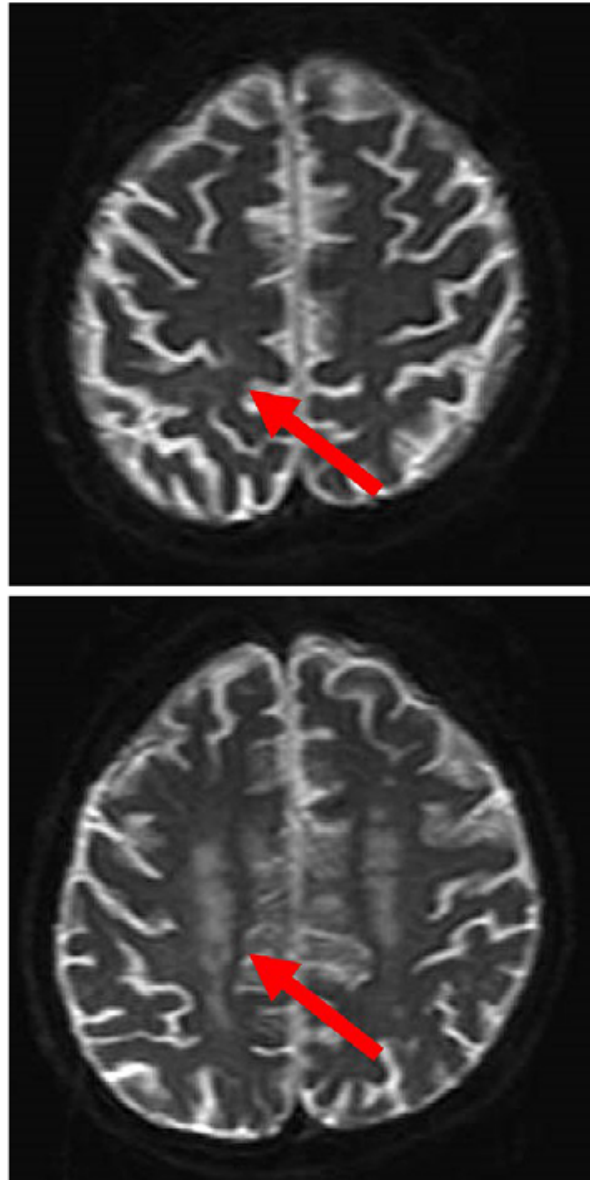

Figure 6. Head MRI finding at the second admission. Head MRI revealed high signal intensity on the diffusion weighted image (DWI) and low signal intensity on the apparent diffusion coefficient (ADC) map in the right hemisphere, which indicated acute lacunar infarction. phase DIC diagnostic criteria of the patient was 6 points. ${ }^{6}$ The main cause of DIC was heat stroke; according to the guidelines, ${ }^{7)}$ heat stroke is the most serious condition in heat-related illness and is defined as patients exposed to high environmental temperature who meet one or more of the following criteria: central nervous system manifesta- tions, hepatic/renal dysfunction, and coagulation disorder. The situation in which the patient laid down in a room without air-conditioning in the middle of summer and could not drink enough water could explain the exposure to a high environmental temperature. Moreover, consciousness impairment, hepatic and renal dysfunction, and 

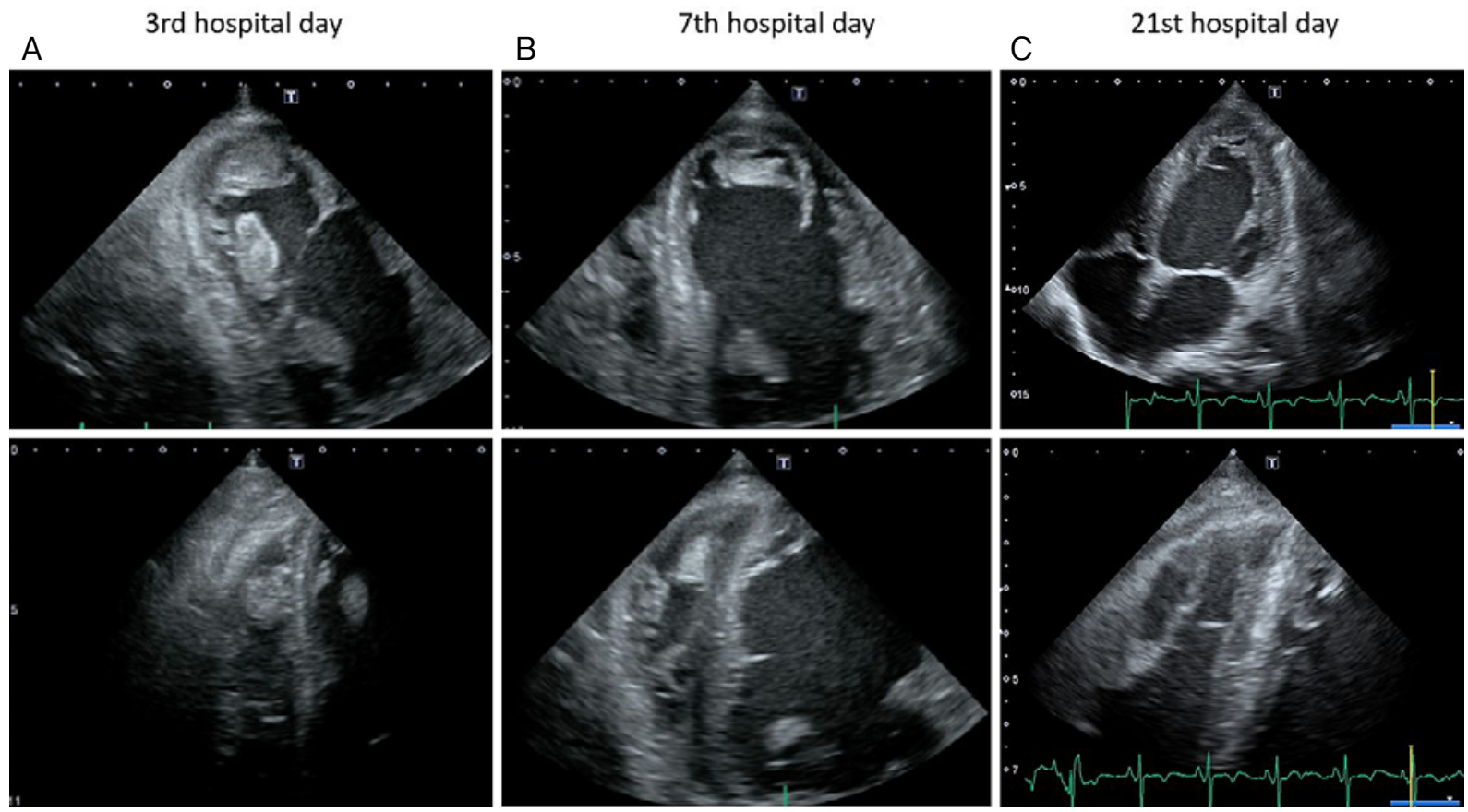

Figure 7. The transition of biventricular thrombi in TTE findings. The upper row shows the left ventricle and the lower row shows the right ventricle. A: The left column indicates the findings obtained on the 3rd hospital day. B: The middle column indicates the findings obtained on the 7 th hospital day. C: The right column indicates the findings obtained on the 21 st hospital day. The ventricular thrombi tended to diminish with time.

DIC were observed in the patient at the second admission, all of which were consistent with heat stroke. These combined conditions led to thrombus formation (Supplemental Figure 4), and consequently, thrombi were formed in the right and left ventricles. There were no findings indicating connective tissue disease, malignancy, or hematologic disease, which causes hypercoagulability, from blood tests (Supplemental Table) and plain CT.

In general, ventricular thrombosis is most often discovered in the left ventricle, particularly after MI. RV thrombosis is uncommon in the general population, and biventricular thrombi are extremely rare. In this case, systolic dysfunction and ventricular dilatation were demonstrated in both the left and right ventricles, which may have also affected the formulation of thrombi in both ventricles. Although it was sufficient to evaluate LV function with 2-dimensional (2-D) TTE, it was difficult to estimate RV function accurately using this method. RV systolic dysfunction and the tendency of RV dilatation in this case were assessed by the appearance on 2-D TTE. It should be noted that the skills and ability of the observer had a considerable impact on the successful evaluation of RV function using TTE. Quantitative indices in 2-D TTE such as tricuspid annular plane systolic excursion and fractional area change were not measured for accurate assessment of $\mathrm{RV}$ function in this case. Therefore, we believe that RV dysfunction could be related to the formation of $\mathrm{RV}$ thrombi on the qualitative assessment, but no quantitative assessment was performed to confirm this relationship. Other modalities such as 3-D TTE and cardiac magnetic resonance (CMR) could be important and useful in this context. $^{8)}$
According to the domestic reports on biventricular thrombi, ${ }^{9)}$ only 15 cases have been reported so far, with a similar tendency observed abroad. When we searched the terms "biventricular" and "thrombus" in PubMed, only 30 cases were found to have been reported, none of which reported biventricular thrombi associated with systolic dysfunction and DIC from heat stroke. Most cases of biventricular thrombi present with systolic dysfunction; the primary cause of which was dilated cardiomyopathy, followed by peripartum cardiomyopathy and MI. Other causes include myocarditis and drug-induced cardiomyopathy, and cases of preserved EF have also been published. For example, when a patient contracts nephrotic syndrome or has cocaine disorder, which induces a hypercoagulable state. ${ }^{10,11)}$ In this context, any factor of Virchow's triad may promote the production of biventricular thrombi, and patients with heart failure in clinical settings should be considered for the derivatives of thrombus. In particular, cases of DIC caused not only by heat stroke but also as a result of acute leukemia or sepsis require careful attention for the formation of thrombi.

There are currently no helpful guidelines in the literature for the assessment of the treatment for biventricular thrombi. The ESC guideline recommends the use of oral anticoagulation for up to 6 months in patients with a single LV thrombus after an ST-segment elevation MI. ${ }^{12}$ In previous reports, the mainstream therapy for biventricular thrombi was anticoagulation with heparinization, and only 2 of the 7 cases of dilated cardiomyopathy were operated on to remove the thrombi, ${ }^{2,13}$ both cases had mobile thrombi, and one resulted in complications of renal infarction. Given these experiences, we suggest that mobility 
and embolism are key factors in determining thrombus removal. Although it is difficult to assess mobility objectively, the findings of TTE are very useful, and clinicians should carefully observe the movement of the thrombus using this technique. Meanwhile, MRI and contrast CT should be utilized for the evaluation of embolism. In this case, we cautiously discussed the indications for the operation, and anticoagulation therapy was selected for 3 reasons. First, the patient was in a serious condition at the time of admission, and was consequently unable to receive the invasive intervention because of multiple organ failure and DIC. Second, the thrombi motility was not ascertained using TTE; there were no thrombi necks, and the spherical thrombi contacted and shared borders with the intraventricular wall. Further, the echoic brightness of all thrombi was high, which were unlikely to be fresh thrombi or travel to the peripheral arteries. Lastly, the apparent embolism was not confirmed on plain CT. Certainly, head MRI showed right hemisphere lacunar infarction; however, this infarction did not indicate cardiogenic infarction at the point of the infarction region and distribution. In the case of cardiogenic infarction, magnetic resonance angiography (MRA) would typically show the occlusion of a major cerebral artery and the relatively broad infarction region; however, these findings were not obtained in this case. Consequently, internal anticoagulation therapy was selected, and the patient never encountered a pulmonary embolism or systematic embolism due to dissemination of the thrombus.

It is also important to consider systolic cardiac dysfunction. The current patient may have been affected by alcoholic cardiomyopathy, although dilated cardiomyopathy could not be denied. In TTE findings at the initial presentation, the EF was $5.9 \%$. Following the treatment, cardiac function improved to $30.7 \% 2$ months after discharge. However, the consecutive alcohol ingestion after the first hospitalization and the interruption of medications since 3 weeks before the second admission made his cardiac function worsen on the second admission. In fact, the EF reversed to $15 \%$ at the second admission. The significant increase in NT-pro BNP was likely to be predominantly due to congestive heart failure, but the hemoconcentration may have also affected the elevation of NT-pro BNP. During the second hospitalization, the medication therapy including the introduction of $\beta$-blockers and the discontinuation of alcohol ingestion may have contributed to the improvement in systolic function. The latest finding of TTE revealed the amelioration of EF to $45 \%$. Relating to the cause of systolic dysfunction, ischemic cardiomyopathy was unlikely because coronary CT scans showed no severe coronary stenosis. Prior to hospitalization, he regularly consumed 52.5-70 g alcohol per day. Alcoholic cardiomyopathy is caused by consuming more than $80 \mathrm{~g}$ of alcohol per day for at least 5 years. ${ }^{14,15)}$ Considering that his body weight was under $50 \mathrm{~kg}$, the amount of his ingested alcohol was enough for him to suffer from alcoholic cardiomyopathy. If drinking alcohol is limited after discharge, cardiac function tends to improve, although LV reverse remodeling as a consequence of treatment with $\beta$-blockers also contributes to cardiac function recovery. Moreover, the fact that alcoholic cardi- omyopathy induces dilation of both the left and right ventricles is consistent with the biventricular systolic dysfunction in this case. ${ }^{16,17)}$ Based on these reasons, we believe that alcoholic cardiomyopathy is most likely. Beriberi heart could have coexisted due to vitamin B1 deficiency, although vitamin B1 was not measured at admission. In general, the beriberi heart is a high-output heart failure. In terms of heart failure with reduced EF and dry-cold findings in the Nohria/Stevenson classification, which indicates low output heart failure, beriberi heart was not the main cause of heart failure in the current case, but may have an effect on the onset of heart failure. CMR will provide more helpful information on the cause of systolic dysfunction in the future.

The difference in ECGs between the first and second admissions was not clearly explained, but may imply the progression of myocardial injury associated with cardiomyopathy. Even if an ST-segment change or giant negative $\mathrm{T}$ wave was obtained, ischemic heart disease or Takotsubo cardiomyopathy would have been considered as a differential diagnosis. However, these findings were not present, and the inverted $\mathrm{T}$ wave in $\mathrm{I}, \mathrm{aVL}$, and leads V5 and V6 resulted in an improvement to normal $\mathrm{T}$ waves 1 year later. This change may represent the recovery from myocardial injury due to the abstinence of alcohol.

In the current report, we describe a rare case of biventricular thrombi and complete disappearance of thrombi without surgical intervention. No previous studies have analyzed the frequency, complications, or mortality of biventricular thrombi. Assessment of similar cases, together with robust statistical analysis and definition of the indications for surgical intervention, are required in the future.

\section{Conclusion}

This case represents biventricular thrombi triggered by DIC from heat stroke in combination with cardiac systolic dysfunction. To the best of our knowledge, a case of biventricular thrombi that were formed by this pathophysiology has not been previously reported. The patient was treated with internal anticoagulation therapy without surgical intervention, and no complications have occurred. For similar cases, in the absence of thrombus mobility and infarction, the complete disappearance of the thrombus may be achievable with conservative therapy alone.

\section{Disclosure}

Conflicts of interest: None.

\section{References}

1. Silvia G, Abdel G, Ioannis D, et al. Incidence and predictors of early left ventricular thrombus after ST-elevation myocardial infarction in the contemporary era of primary percutaneous coronary intervention. Am J Cardiol 2014; 113: 1111-6.

2. Iwano T, Yunoki K, Tokunaga $\mathrm{N}$, et al. A case of biventricular thrombi in a patient with dilated cardiomyopathy: Utility of multimodality imaging for diagnosis and management of treatment strategy. J Cardiol Cases 2017; 15: 91-4. 
3. Dickson BC. Venous thrombosis: on the history of Virchow's Triad. Univ Toronto Med J 2004; 81: 166-71.

4. Zakauddin AV, Carroll EC. Cardiovascular alterations in hea stroke. Chest 1993; 103: 987-8.

5. Watanabe C, Nishina N, Kawai C. Stress-induced cardiomyopathy accompanied by heat stroke. J Cardiol Case 2015; 12: 16-9.

6. Gando S, Matoba T, Eguchi Y, et al. JAAM DIC diagnostic criteria. J Jpn Assoc Acute Med 2005; 16: 188-202 (Japanese).

7. Okudera H, Kitahara T, Shimazaki S, et al. Final report of heatstroke study Japanese Association for Acute Medicine. J Jpn Assoc Acute Med 2014; 25: 846-62 (Japanese).

8. Sara L. Partington, Philip J. Kilner. How to Image the Dilated Right Ventricle. Circ Cardiovasc Imaging 2017; 10: e004688.

9. Kitazono I, Yamashita M, Motodaka H, et al. A resected case of biventricular thrombi with cardiac sarcoidosis. Jpn J Cardiovasc Surg 2007; 36: 261-4.

10. Huang TY, Chau KM. Biventricular thrombi in diabetic nephrotic syndrome complicated by cerebral embolism. Int. J Cardiol 1995; 50: 193-6.

11. Azadeh G, Juma B, Antonio L. Acute renal infarction, transient ischemic attack, and biventricular thrombi secondary to substance use disorder: A case report. Clin Case Rep 2019; 7: 8614.

12. Ibanez B, James S, Agewall S, et al. 2017 ESC Guidelines for the management if acute myocardial infarction in patients presenting with ST-segment elevation: The Task Force for the man- agement of acute myocardial infarction in patients presenting with ST-segment elevation of the European Society of Cardiology. Eur Heart J 2018; 39: 119-77.

13. Hatanaka N, Ueda T. Removal of biventricular thrombi for dilated cardiomyopathy in a patient with acute heart failure. Kyobu Geka 2014; 67: 895-8 (Japanese).

14. Prazak P, Pfisterer M, Osswald S, Buser P, Burkart F. Differences of disease progression in congestive heart failure due to alcoholic as compared to idiopathic dilated cardiomyopathy. Eur Heart J 1996; 17: 251-7.

15. Antonio M, Claudia T, Gabriele A, et al. Alcoholic cardiomyopathy: What is known and what is not known. Eur $\mathrm{J}$ Int Med 2017; 43: 1-5.

16. Kryzhanovskii SA, Kolik LG, Tsorin IB, et al. Alcoholic Cardiomyopathy. Bull Exp Biol Med 2017; 5: 627-31.

17. Kajander OA, Kupari M, Laippala P, et al. Dose dependent but non-linear effects of alcohol on the left and right ventricle. Heart 2001; 86: 417-23.

\section{Supplemental Files}

Supplemental Table

Supplemental Figures 1-4

Please see supplemental files; https://doi.org/10.1536/ihj.20-558 